\title{
Interações medicamentosas em fitoterápicos
}

\author{
Drug interactions in herbal medicines \\ Interacciones farmacológicas en medicamentos a base de hierbas
}

Recebido: 05/11/2021 | Revisado: 12/11/2021 | Aceito: 15/11/2021 | Publicado: 17/11/2021

\author{
Michele Cristina da Silva \\ ORCID: https://orcid.org/0000-0002-9821-7544 \\ Centro Universitário UNIFAVIP/WYDEN, Brasil \\ E-mail: michelecristina208@gmail.com \\ Priscilla da Silva Colino \\ ORCID: https://orcid.org/0000-0003-1463-586X \\ Centro Universitário UNIFAVIP/WYDEN, Brasil \\ E-mail: cillacolino@hotmail.com \\ João Gomes Pontes Neto \\ ORCID: https://orcid.org/0000-0001-9294-9448 \\ Centro Universitário UNIFAVIP/WYDEN, Brasil \\ E-mail: joao.neto1@unifavip.edu.br
}

\begin{abstract}
Resumo
Desde as primeiras civilizações, o homem, de forma empírica, buscava o alívio e a cura para as enfermidades que os acometiam. Descobriram nas plantas atividades medicinais e terapêuticas, passando a utilizar esse recurso para tratar as mais diversas doenças de acordo com o conhecimento adquirido no decorrer dos tempos. O medicamento fitoterápico é aquele obtido com uso exclusivo de matérias-primas vegetais, ou seja, ativos extraídos de plantas, ou parte específica que contém a atividade terapêutica. O objetivo do presente estudo é expor e confrontar informações sobre interações medicamentosas dentro da fitoterapia, tendo em vista que ainda há, na população, uma certa crença de que medicamentos derivados de plantas não apresentam eventos adversos. Trata-se de um estudo de revisão de literatura, onde os dados obtidos reforçam a importância desse tipo de informação para a sociedade, que cada vez mais busca nos medicamentos naturais alternativas para melhoria da saúde e longevidade. A pesquisa envolveu artigos entre 2008 e 2021, onde foi observado que há relatos significativos de interações medicamentosas associadas ao uso de fitoterápicos. Os dados levantados são de extrema importância para quebrar tabus relacionados à segurança dos medicamentos de origem natural, contribuindo para seu uso racional.
\end{abstract}

Palavras-chave: Plantas medicinais; Interações medicamentosas; Fitoterápicos; Terapêutica.

\begin{abstract}
Since the first civilizations, man, empirically, sought relief and cure for the diseases that afflicted them. They discovered medicinal and therapeutic activities in plants, starting to use this resource to treat the most diverse diseases according to the knowledge acquired over time. The herbal medicine is that obtained with the exclusive use of vegetable raw materials, that is, actives extracted from plants, or a specific part that contains the therapeutic activity. The aim of this study is to expose and compare information about drug interactions within herbal medicine, considering that there is still a certain belief in the population that herbal medicines do not present adverse events. This is a literature review study, where the data obtained reinforce the importance of this type of information for society, which increasingly seeks alternative natural medicines to improve health and longevity. The research involved articles between 2008 and 2021, where it was observed that there are significant reports of drug interactions associated with the use of herbal medicines. The data collected are extremely important to break taboos related to the safety of medicines of natural origin, contributing to their rational use.
\end{abstract}

Keywords: Medicinal plants; Drug interactions; Herbal medicines; Therapy.

\section{Resumen}

Desde las primeras civilizaciones, el hombre, empíricamente, buscó alivio y curación de las enfermedades que lo aquejaban. Descubrieron actividades medicinales y terapéuticas en las plantas, comenzando a utilizar este recurso para tratar las más diversas enfermedades de acuerdo con los conocimientos adquiridos a lo largo del tiempo. La fitoterapia es aquella obtenida con el uso exclusivo de materias primas vegetales, es decir, activos extraídos de plantas, o una parte específica que contenga la actividad terapéutica. El objetivo de este estudio es exponer y comparar información sobre las interacciones medicamentosas dentro de la fitoterapia, considerando que aún existe una cierta creencia en la población de que las medicinas herbales no presentan eventos adversos. Se trata de un estudio de revisión de la literatura, donde los datos obtenidos refuerzan la importancia de este tipo de información para la sociedad, que busca cada vez más medicinas naturales alternativas para mejorar la salud y la longevidad. La investigación involucró 
artículos entre 2008 y 2021, donde se observó que existen informes significativos de interacciones medicamentosas asociadas con el uso de medicamentos a base de hierbas. Los datos recopilados son de suma importancia para romper tabúes relacionados con la seguridad de los medicamentos de origen natural, contribuyendo a su uso racional.

Palabras clave: Plantas medicinales; Interacciones con las drogas; Hierbas medicinales; Terapia.

\section{Introdução}

Fitoterápicos são medicamentos compostos exclusivamente por substâncias extraídas de matéria-prima vegetal, podendo ser sólido ou líquido. Na forma liquida, podem ser preparados através de etanol e água, xaropes, tinturas e óleos medicinais. Na forma sólida, os solventes são evaporados e a matéria-prima resultante pode ser utilizada para produção de medicamentos na forma de pó, grânulos ou comprimidos (Aleluia et al., 2017).

Nessa perspectiva existem vários tipos de fitoterápicos. Os mais consumidos pela população são 15: Hedera helix, Valeriana officinalis, Passiflora incarnata, Ginkgo bilola, Ananos comosur, Equisetum, Lipindium meyenii, Maytenus ilicifolia, Spirulina, Harpagophytum, Procumbens, Cúrcuma longa, Mikania glomarata, Senna alexandrina, Centella asiática. Os fitoterápicos utilizados para emagrecimento, calmantes, expectorantes e anti-inflamatórios foram destaque no consumo (Silva et al., 2018).

As interações medicamentosas são divididas em farmacodinâmicas e farmacocinéticas. As interações farmacodinâmicas podem acontecer devido ao princípio ativo ou por seus metabolitos, podendo se apresentar como uma sinergia ou antagonismo. Já as interações farmacocinéticas estão relacionadas a alterações na absorção ou transformação metabólica dos ativos (Gelatti et al., 2016). Por exemplo, o extrato de Ginkgo usado como estimulante de memória e contra vertigem, também é capaz de potencializar a ação do ácido acetilsalicílico, do clopidogrel, anticoagulantes e de antiinflamatórios não-asteroidais de forma que haja um alto risco de sangramento (Carvalho \& Rocha, 2016).

Ferreira (2019) escreve que o medicamento derivado da espécie Valeriana officinalis, é um fitoterápico utilizado como sedativo moderado e auxilia ao tratamento de distúrbio do sono relacionado à ansiedade. As interações com as classes farmacológicas de benzodiazepínicos, ansiolíticos e hipnóticos, como alprazolam e midazolam, resultam em sinergismo e seus efeitos terapêuticos são potencializados na depressão do Sistema Nervoso Central (SNC).

Por sua vez Sá et al. (2018) esclarece que os fitoterápicos são definidos por vários constituintes químicos, possuem diversos mecanismo de ação e apresentam interações com diversos medicamentos, influenciando na eficácia e segurança dos fármacos. Por existir a possibilidade de interações medicamentosas entre fitoterápicos e fármacos e por haver uma certa crença popular de que medicamentos naturais não apresentam eventos adversos, a presente pesquisa se mostra extremamente necessária para difundir informações que possam esclarecer a população sobre o uso racional de fitoterápicos.

O objetivo do presente estudo é expor e confrontar informações sobre interações medicamentosas dentro da fitoterapia, tendo em vista que ainda há, na população, uma certa crença de que medicamentos derivados de plantas não apresentam eventos adversos.

\section{Metodologia}

Trata-se de uma pesquisa bibliográfica do tipo Revisão Integrativa (RI), classificada como uma pesquisa de natureza básica, abordagem Qualitativo-Quantitativa e quanto aos objetivos Exploratória. Na primeira etapa foram realizadas a seleção dos artigos, utilizando como pergunta de pesquisa, dessa forma formulou-se a seguinte questão da pesquisa: Quais fitoterápicos fazem interações medicamentosas? A RI possibilita a análise, identificação de diversos estudos dentro uma mesma temática e a síntese do conhecimento, contribuindo para a criação de um panorama organizado e firme de conceitos do interesse do estudo (Souza et al., 2010).

Para a busca dos artigos, foram utilizadas as seguintes palavras chaves: "Plantas Medicinais", "Interações 
Medicamentosas", "Interações Planta-Droga", "Interações Adversas", "Interações Benéficas". Também foi utilizado o operador booleano "AND” para associá-las. Como critério para a seleção dos artigos foram incluídos todos os artigos com resumos e textos completos disponíveis para análise, no idioma português entre os anos 2008 e 2021 e que respondessem à pergunta norteadora. Foram excluídas as publicações que não estavam no formato de artigo científico, como livros, resenhas, cartas e editoriais.

Os levantamentos dos artigos na literatura foram realizados nas bases de dados: Scielo, Biblioteca Virtual em Saúde: BVS, LILACS e Google Acadêmico. O presente estudo por não se tratar de uma pesquisa, e sim revisão, não foi submetido à avaliação do Comitê de Ética, e cumpriu os padrões éticos estabelecidos para garantir que as legalidades das informações estejam de acordo com o padrão estabelecido, não foi envolvido a participação de seres humanos, todas as realizações utilizadas nesse trabalho serão devidamente referenciadas de acordo com a norma APA.

\section{Resultados e Discussão}

O resultado das buscas permitiu identificar pesquisas voltadas para avaliação das interações medicamentosas dos fitoterápicos. A busca nas bases de dados, utilizando os cruzamentos em dupla, resultou em 396 artigos que, após leitura do título e resumo, 22 publicações foram identificadas inicialmente, conforme apresentado na Tabela 1.

Tabela 1. Estratégias de busca e número de manuscritos obtidos nos recursos informacionais. Caruaru, PE, Brasil, 2021.

\begin{tabular}{llll}
\hline $\begin{array}{l}\text { Recurso } \\
\text { informacional }\end{array}$ & Estratégia de Busca & $\begin{array}{l}\text { Localizados na } \\
\text { busca }\end{array}$ & $\begin{array}{l}\text { Selecionados por título e } \\
\text { resumo }\end{array}$ \\
\hline Scielo & $\begin{array}{l}\text { Interações medicamentosas de } \\
\text { fitoterápicos } \\
\text { Interações medicamentosas de } \\
\text { fitoterápicos } \\
\text { Interações medicamentosas de } \\
\text { filoterápicos }\end{array}$ & 6 & 3 \\
Google acadêmico & & 368 & 1 \\
TOTAL & & 395 & 14 \\
\hline
\end{tabular}

Fonte: Autores (2021).

Posteriormente, foi feita a exclusão de 4 publicações duplicadas, restando 18 artigos que foram lidos na íntegra. Esses 18 estudos compuseram a amostra para ser avaliada na análise final. Uma vez que 8 artigos foram excluídos por não corresponderam aos critérios de inclusão, restaram 10 trabalhos para serem analisados. 
Figura 1. Fluxograma do processo de identificação, seleção e inclusão dos estudos adaptado do PRISMA.

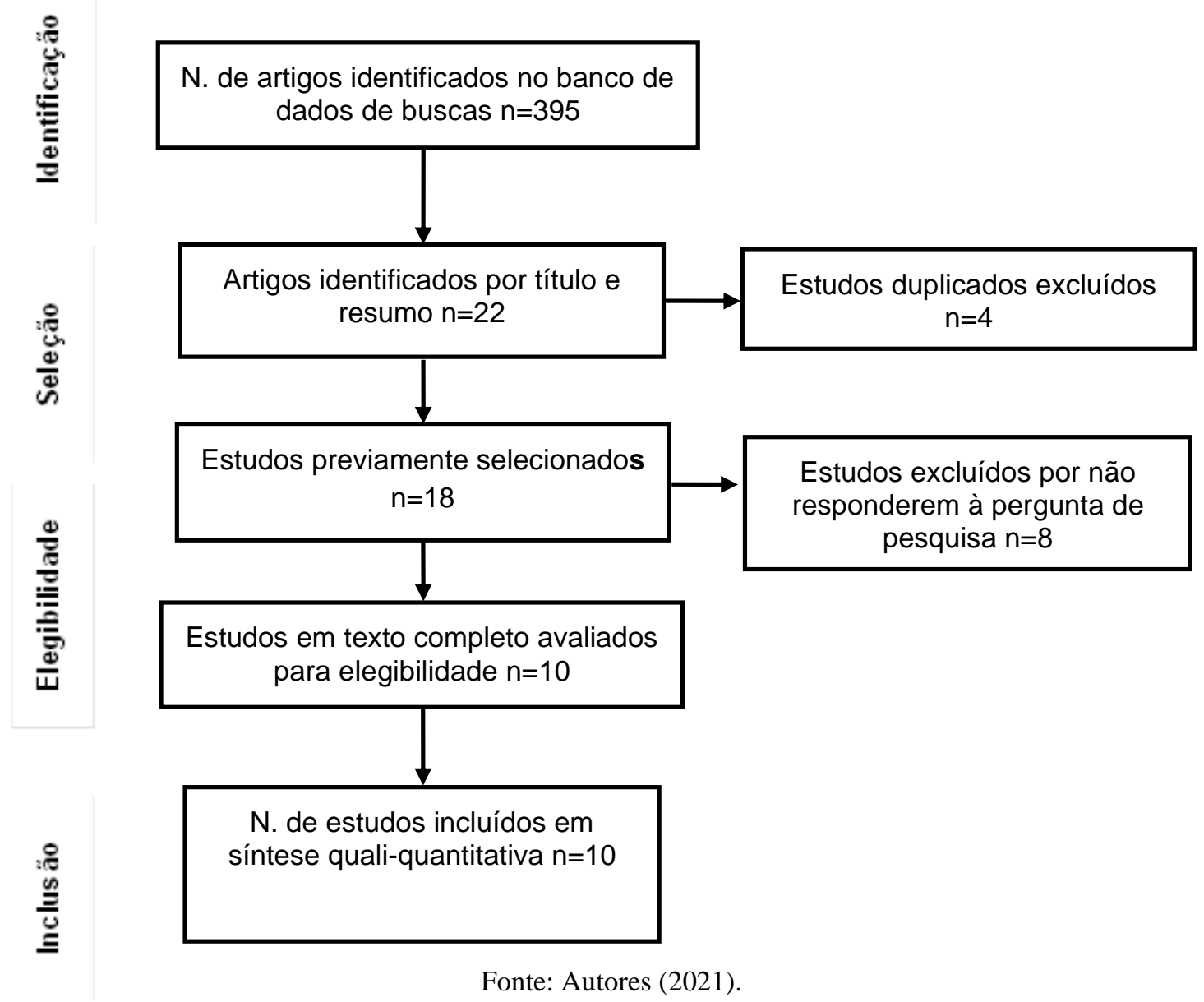

A maioria das publicações ocorreram nos anos 2008 e 2016. Observa-se que se tem poucas publicações recentes dentro dessa temática, demonstrando a necessidade de mais estudos. Posteriormente foi realizada a identificação do desenho do estudo utilizado nos artigos, verificando-se que $40 \%$ são estudos de RI. A distribuição dos estudos selecionados segundo o desenho está presente na tabela 2.

Tabela 2. Distribuição dos estudos identificados nas bases de dados segundo características metodológicas, no período 2008 e 2021.

\begin{tabular}{lcc}
\hline TIPO DE ESTUDO & NÚMERO DE ESTUDO & PORCENTAGEM (\%) \\
\hline Revisão Integrativa & $\mathbf{4}$ & $\mathbf{4 0 \%}$ \\
\hline Estudo transversal & $\mathbf{2}$ & $\mathbf{2 0 \%}$ \\
\hline $\begin{array}{l}\text { Questionário fechado e } \\
\text { entrevista }\end{array}$ & $\mathbf{3}$ & $\mathbf{3 0 \%}$ \\
\hline $\begin{array}{l}\text { Descritiva por } \\
\text { porcentagem }\end{array}$ & $\mathbf{1}$ & $10 \%$ \\
\hline TOTAL & & $\mathbf{1 0 0 \%}$ \\
\hline
\end{tabular}


Observou-se que os estudos, majoritariamente, se tratava de revisões. A RI é uma coordenação para pesquisar e examinar artigos de uma determinada área da ciência. Com a importância de definir a linha mais próxima da pesquisa que pretende descrever, é preciso constituir os tópicos chave, autores, palavras, periódicos e fontes de dados preliminares, tem caráter exploratório, tem mais afinidade com o problema exposto (Conforto et al., 2011).

Quadro 1. Caracterização dos artigos selecionados para análise segundo autores, título, local, fitoterápicos, medicamentos e interações no período 2008-2021.

\begin{tabular}{|c|c|c|c|c|}
\hline AUTORES & TÍTULO & FITOTERAPICOS & $\begin{array}{c}\text { CLASSE } \\
\text { TERAPEUTICA }\end{array}$ & INTERAÇÕES \\
\hline Silva (2016) & $\begin{array}{l}\text { Tudo que é natural não faz } \\
\text { mal? Investigação sobre o } \\
\text { uso de plantas medicinais e } \\
\text { medicamentos fitoterápicos } \\
\text { por idosos, na cidade de } \\
\text { Iapu- leste de Minas Gerais }\end{array}$ & $\begin{array}{c}\text { Uncaria tomentosa } \\
\text { Aesculus } \\
\text { hippocastanum } \\
\text { Centelha asiática } \\
\text { Thuja accidentais }\end{array}$ & $\begin{array}{l}\text { Anti hipertensivo } \\
\text { Anticoagulantes } \\
\text { Insulina } \\
\text { Carbamazepina }\end{array}$ & $\begin{array}{l}\text { Aumenta o potencial do fármaco. } \\
\text { Potencializa o risco de hemorragia } \\
\text { cerebral. } \\
\text { Diminui o efeito da glibenclamida e } \\
\text { metformina. } \\
\text { Reduz o limiar convulsivo. }\end{array}$ \\
\hline $\begin{array}{l}\text { Lorenz, Klein, } \\
\text { Colet, Armador e } \\
\text { Heineck (2016) }\end{array}$ & $\begin{array}{c}\text { O uso de plantas medicinais } \\
\text { e fitoterápicos em usuários } \\
\text { de varfarina no município de } \\
\text { Ijuí/RS }\end{array}$ & $\begin{array}{c}\text { Camellia sinesis } \\
\text { Mikania glomerata }\end{array}$ & $\begin{array}{l}\text { Varfarina } \\
\text { Varfarina }\end{array}$ & $\begin{array}{c}\text { Aumenta o risco de tromboembolia, } \\
\text { Grande quantidade de vitamina k que } \\
\text { atua como antagonista diminui os valores } \\
\text { do INR. } \\
\text { Apresenta cumarias na sua, composição } \\
\text { desenvolvendo acidentes hemorrágicos, } \\
\text { pelo antagonismo à vitamina k. }\end{array}$ \\
\hline $\begin{array}{l}\text { Nicacio, Pinto, } \\
\text { Silva, Silva, } \\
\text { Matos e Goulart } \\
\quad \text { (2016) }\end{array}$ & $\begin{array}{l}\text { Potenciais interações entre } \\
\text { medicamentos alopáticos e } \\
\text { fitoterápicos/ plantas } \\
\text { medicinais no Município de } \\
\text { Rondonópolis - MT }\end{array}$ & $\begin{array}{l}\text { Passiflora incarnata } \\
\text { Ginkgo biloba } \\
\text { Valleriana officinalis }\end{array}$ & $\begin{array}{c}\text { Cinarizina } \\
\text { Atenalol } \\
\text { Amitriptílina }\end{array}$ & $\begin{array}{c}\text { Aumenta depressão do SNC 50\%. } \\
\text { Bradicardia e hipotensão 25\%. } \\
\text { Aumenta depressão do SNC. }\end{array}$ \\
\hline $\begin{array}{l}\text { Souza e Silva } \\
\text { (2018) }\end{array}$ & $\begin{array}{l}\text { Chás e fitoterápicos } \\
\text { indicados para distúrbios do } \\
\text { sono, ansiedade e depressão, } \\
\text { disponibilizados em } \\
\text { estabelecimentos comerciais } \\
\text { de São Caetano do Sul - SP. }\end{array}$ & $\begin{array}{l}\text { Passiflora incarnata } \\
\text { Hypericum perforatum }\end{array}$ & $\begin{array}{l}\text { Benzodiazepínicos, } \\
\text { barbitúricos } \\
\text { Ciclosporina } \\
\text { Anticoagulantes } \\
\text { Cumarínicos } \\
\text { Anticoncepcional }\end{array}$ & $\begin{array}{l}\text { Aumenta a depressão do SNC. } \\
\text { Diminui os níveis séricos e eficácia } \\
\text { terapêutica. }\end{array}$ \\
\hline $\begin{array}{l}\text { Riboldi e Rigo } \\
\text { (2019) }\end{array}$ & $\begin{array}{c}\text { Análise do uso de plantas } \\
\text { medicinais e medicamentos } \\
\text { em habitantes do município } \\
\text { de Capitão/RS }\end{array}$ & $\begin{array}{l}\text { Matrícaria recutita } \\
\text { Mentha piperito } \\
\text { Capim-limão } \\
\text { Funcho }\end{array}$ & $\begin{array}{c}\text { Aas, heparina, } \\
\text { anticoagulantes, } \\
\text { Barbitúricos e b } \\
\text { Benzodiazepínicos } \\
\text { Sinvastatina } \\
\text { Felodipina } \\
\text { Clonazepam } \\
\text { Diazepam } \\
\text { Omeprazol }\end{array}$ & $\begin{array}{l}\text { Aumenta o risco de hemorragia. } \\
\text { Pode potencializar o efeito sedativo } \\
\text { desses fármacos. } \\
\text { Óleo dessas plantas interfere nas enzimas } \\
\text { do citocromo P450 podendo elevar o } \\
\text { nível dos fármacos intensicando seus } \\
\text { efeitos. } \\
\text { Podendo aumentar o efeito dos fármacos. } \\
\text { Furanocumarinas podem inibir a } \\
\text { atividade de enzimas de metabolização } \\
\text { CIP3A4 e CIP2D6. }\end{array}$ \\
\hline $\begin{array}{l}\text { Dias, Trevisan, } \\
\text { Nagai, Ramos e } \\
\text { Silva (2017) }\end{array}$ & $\begin{array}{l}\text { Uso de fitoterápicos e } \\
\text { potenciais riscos de } \\
\text { interações medicamentosas: } \\
\text { reflexões para prática segura }\end{array}$ & $\begin{array}{l}\text { Hypericum perforatum } \\
\text { L. } \\
\text { Matricaria recutita L. } \\
\text { Cynara scolymus } \\
\text { Glycine may L.Merr }\end{array}$ & $\begin{array}{c}\text { Etinilestradiol } \\
\text { Ciclosporina } \\
\text { Fenobarbital } \\
\text { Furosemida } \\
\text { Hidrocloratiazida } \\
\text { levotiroxina }\end{array}$ & $\begin{array}{l}\text { Aumenta o metabolismo hormonal com } \\
\text { sangramento menstrual. } \\
\text { Diminui a concentração plasmáticas do } \\
\text { fármaco e risco de rejeição de } \\
\text { transplantes. } \\
\text { Aumenta o prolongamento da ação } \\
\text { depressora do SNC. Diminui } \\
\text { o volume sanguíneo e queda de pressão }\end{array}$ \\
\hline
\end{tabular}




\begin{tabular}{|c|c|c|c|c|}
\hline & & & & $\begin{array}{c}\text { arterial hipocalemia. } \\
\text { Baixa absorção do fármaco. }\end{array}$ \\
\hline $\begin{array}{c}\text { Alexandre, } \\
\text { Bagatini e } \\
\text { Simões (2008) }\end{array}$ & $\begin{array}{l}\text { Uso de fitoterápicos e } \\
\text { potenciais riscos de } \\
\text { interações medicamentosas: } \\
\text { reflexões para prática segura }\end{array}$ & $\begin{array}{l}\text { Valeriana officinalis } \\
\text { Allium sativum }\end{array}$ & $\begin{array}{l}\text { Alprazolam } \\
\text { Midazolam } \\
\text { Tiopental } \\
\text { Pentobarbital } \\
\text { Loperamida } \\
\text { Paracetamol } \\
\text { Clopropomida } \\
\text { Lisinopril }\end{array}$ & $\begin{array}{c}\text { Aumenta os efeitos terapêuticos e da } \\
\text { depressão do SNC. } \\
\text { Delírios, confusão, agitação e } \\
\text { desorientação } \\
\text { Alterações nos perfis farmacocinéticos } \\
\text { dos } \\
\text { Fármacos. } \\
\text { Hipoglicemia } \\
\text { Aumenta o efeito do fármaco }\end{array}$ \\
\hline $\begin{array}{c}\text { Marliére, } \\
\text { Ribeiro, } \\
\text { Brandão, Klein e } \\
\text { Acurcio (2008) }\end{array}$ & $\begin{array}{l}\text { Utilização de fitoterápicos } \\
\text { por idosos: resultados de um } \\
\text { inquérito domiciliar em Belo } \\
\text { Horizonte (MG), Brasil }\end{array}$ & $\begin{array}{c}\text { Aesculus } \\
\text { hippocastanum } \\
\text { Cratengo }\end{array}$ & $\begin{array}{c}\text { Ticlopidina } \\
\text { Hidrocloratiazida }\end{array}$ & $\begin{array}{l}\text { Aumenta o efeito antiagregante } \\
\text { Aumento da pressão arterial }\end{array}$ \\
\hline $\begin{array}{c}\text { Alexandre, } \\
\text { Bagatini e } \\
\text { Simões (2008) }\end{array}$ & $\begin{array}{l}\text { Interações entre fármacos e } \\
\text { medicamentos fitoterápicos à } \\
\text { base de ginkgo ou ginseng }\end{array}$ & Ginkgo biloba L. & $\begin{array}{l}\text { Varfarina } \\
\text { Ibuprofeno } \\
\text { Omeprazol } \\
\text { Nifedipina } \\
\text { Fluoxetina }\end{array}$ & $\begin{array}{c}\text { Aumenta o risco de hemorragia } \\
\text { Aumenta o risco de hemorragia. } \\
\text { Baixa plasmática e do efeito terapêutico. } \\
\text { Aumenta o efeito adversos do fármaco. } \\
\text { Redução da disfunção sexual }\end{array}$ \\
\hline $\begin{array}{c}\text { Paixão, } \\
\text { Conceição, Neto } \\
\text { e Santos (2016) }\end{array}$ & $\begin{array}{l}\text { Levantamento bibliográfico } \\
\text { de plantas medicinais } \\
\text { comercializadas em feiras da } \\
\text { Bahia e suas interações } \\
\text { medicamentosas }\end{array}$ & $\begin{array}{c}\text { Rosmariuns officinalis } \\
\text { L. } \\
\text { Schinus terebinthifolius } \\
\text { raddi }\end{array}$ & $\begin{array}{c}\text { Diuréticos, laxantes e } \\
\text { hipotensivo } \\
\text { Hipotensores }\end{array}$ & $\begin{array}{l}\text { Distúrbios renais, hipertensão. } \\
\text { Aumenta a ação dos hipotensores. }\end{array}$ \\
\hline
\end{tabular}

Fonte: Autores (2021).

Os resultados dos estudos no período de 2008 a 2021 permitiram visualizar e contextualizar as interações medicamentosas em fitoterápicos que podem estar associadas com a falta de informações a população. No estudo realizado por Silva (2016), avaliando as interações medicamentosas, no período de março a abril de 2012 por meio de um questionário, foram entrevistados 107 idosos no município de Iapu que relataram as interações observadas com maior frequência: os antihipertensivo com a Uncaria tomentosa, Aesculus com anticoagulantes, Hippocastanum com a insulina e a Centelha com carbamazepina.

É fato que os medicamentos fitoterápicos são produzidos à base de princípios ativos de plantas medicinais formados pela mistura de vários outros compostos com efeito terapêutico, porém apesar de suas propriedades benéficas, a utilização do fitoterápico em excesso ou associado à algum fármaco de maneira inadequada pode gerar complicações (David \& Bello, 2017). A utilização de plantas medicinais e, consequentemente fitoterápicos, está fortemente ligada ao aspecto cultural dos povos e que hoje é disseminada mundialmente. Assim, o que antes era apenas sabedoria popular, atualmente é opção terapêutica (D’ávila et al., 2021; Silva \& Leitão, 2021).

Fitoterápicos à base de valeriana são frequentemente indicados em casos de distúrbios do sono, ansiedade, depressão e apresentam uma importante ação sobre pacientes submetidos a estresse ou medo, entretanto tem sido visto que a associação com outros ansiolíticos pode diminuir a expressão de isoformas específicas e, podem desencadear problemas à saúde (Alexandre et al., 2008; Souza \& Silva, 2018). Além disso, seu uso concomitante a antidiarreicos pode causar perturbações mentais como delírios e confusão (D’ávila et al., 2021).

De acordo com a amostra, as pesquisas realizadas nas cidades de São Caetano do Sul - SP (Souza \& Silva, 2018), Capitão - RS (Riboldi \& Rigo, 2019), Belo Horizonte - MG (Marliére et al., 2008) e Iapu - MG (Silva, 2016), evidencia que a 
prevalência no uso de plantas medicinais e fitoterápicos são dos idosos do sexo feminino. Esse dado é de suma relevância, visto que os idosos, na maioria das vezes, utilizam mais de um medicamento, ou classe medicamentosa, continuamente para o tratamento de doenças crônicas. Cerca de $45 \%$ dos idosos avaliados estavam expostos a polifarmácia e à interação medicamentosa entre fármacos e fitoterápicos possuem potenciais riscos à saúde (Marlíere et al., 2008). Os estudos sobre a contraindicação do uso de plantas medicinais e fitoterápicos para idosos são escassos, geralmente contraindica-se o uso para gestantes e crianças (Silva, 2016).

Muitas interações entre fármacos e fitoterápicos ainda são negligenciados ou relatadas como irrelevantes, porém o uso de fitoterápicos à base de Ginkgo associados a diversas classes medicamentosas tem sido descrita na literatura, a exemplo dos anti-hipertensivos e antiflamatórios desencadeando aumento de efeitos adversos, bradicardia, hipotensão e quadros hemorrágicos, já que possui propriedades que pode inibir o fator de ativação plaquetária (Nicácio et al., 2020; Alexandre et al., 2008).

Quanto as interações, merecem uma atenção especial a Rosmariuns officinalis L. e Schinus terebinthifolius raddi em associação à diuréticos e hipotensores devido sua capacidade de desencadear distúrbios renais e prolongar a ação dos hipotensores (Paixão et al., 2016), a Hypericum perforatum L, Matricaria recutita L. Cynara scolymus e a Glycine may L.Merr com os medicamentos Etinilestradiol, Ciclosporina, Fenobarbital, Furosemida, Hidrocloratiazida e Levotiroxina frente ao aumento do metabolismo hormonal, a diminuição da concentração plasmática do fármaco e pode responder negativamente em casos de (Dias et al., 2017). Além disso do aumento da depressão do SNC esteve diretamente relacionado a distintas classes medicamentosas (Dias et al., 2017; Nicácio et al., 2016; Souza \& Silva, 2018; Alexandre et al., 2008).

A literatura demonstra inúmeros relatos de reações desencadeadas por interações entre medicamentos sintéticos e fitoterápicos, apesar de que muitos ainda se mostram inconclusivos. O número de notificações no Sistema Nacional de Notificações para Vigilância Sanitária (NOTIVISA) relacionadas a reações adversas de fitoterápicos ou interações entre medicamentos sintéticos ainda é baixo, indicando uma subnotificação e que pode estar relacionada a impercepção dos efeitos ou riscos ocasionados pelos usuários ou descrédito aos fitoterápicos por considerarem como algo natural que não causa danos (Neves \& Vieira, 2019).

Os fitoterápicos e plantas medicinais mais encontrados na literatura sendo utilizados em associação com medicamentos sintéticos possuem atuação no sistema cardiovascular, sistema nervoso e aparelho digestório (Nicácio et al., 2020). Tendo em vista a atuação dos fitoterápicos e plantas medicinais, seu consumo é feito majoritariamente por automedicação ou por prescrição médica, que na maioria das vezes desconhece os compostos e suas possíveis interações, isso evidencia a deficiência de pesquisas no Brasil sendo direcionada aos principais grupos populacionais (Silveira et al., 2008; Nicácio et al., 2020).

\section{Conclusão}

Os medicamentos fitoterápicos apresentam segurança terapêutica quando orientado e utilizado de maneira correta, caso contrário podem apresentar interações de graus variados quando associado a outros medicamentos, e disparar cascatas de reações causando sinergismo, diminuição ou anulação do efeito de alguns medicamentos e, com isso, o resultado esperado não é alcançado e pode ser comprometido ou mesmo suspenso.

Se faz necessário a ampla divulgação de informações referente a fitoterápicos e interações medicamentosas a fim de minimizar possíveis complicações, visto que os fitoterápicos precisam ser vistos com seriedade baseada em evidências. Ainda, é importante esclarecer a necessidade de políticas públicas de incentivo à disseminação da utilização racional de fitoterápicos, bem como a qualificação dos profissionais de saúde para que possam orientar com efetividade a população. Conclui-se 
também que são necessárias mais pesquisas que possibilitem quebra de paradigmas e mitos em torno dos fitoterápicos associados à medicamentos sintéticos e suas interações.

\section{Referências}

Aleluia, C. D. M., Procópio, V. D. C., Oliveira, M. T. G., Furtado, P. G. S., Giovannini, J. F. G., \& De Mendonça, S. M. S. (2017). Fitoterápicos na Odontologia. Revista de Odontologia da Universidade Cidade de São Paulo, 27(2), 126.

Alexandre, R. F., Bagatini, F., \& Simões, C. M.O (2008). Interações entre fármacos e medicamentos fitoterápicos à base de ginkgo ou ginseng. Revista Brasileira de Farmacognosia, 18, 117-126.

Carvalho, JID, \& Rocha, MSD (2016). Interações Medicamentosas dos Fitoterápicos Ginkgo biloba, Panax ginseng e Hypericum perforatum com medicamentos alopáticos. Centro de Pós-Graduação Oswaldo Cruz.

Conforto, E. C., Amaral, D. C., \& Silva, S. D. (2011). Roteiro para revisão bibliográfica sistemática: aplicação no desenvolvimento de produtos e gerenciamento de projetos. Trabalho apresentado, 8 .

D’ávila, A. M. M. N., de Araújo Cruz, J. H., Guênes, G. M. T., de Oliveira Filho, A. A., \& dos Anjos, R. M. (2021). Interações medicamentosas: fitoterápicos utilizados na Odontologia e fármacos de uso contínuo dos pacientes. Archives of health investigation, 10(3), 468-473.

David, R. B., \& Bello, G. B. (2017). Prescrição de fitoterapia por nutricionistas em farmácias magistrais. BRASPEN (São Paulo), 32(3), $288-92$.

Dias, E. C. M., Trevisan, D. D., Nagai, S. C., Ramos, N. A., \& Silva, E. M. (2017). Uso de fitoterápicos e potenciais riscos de interações medicamentosas: reflexões para prática segura. Revista Baiana de Saúde Pública, 41(2).

Ferreira, F. S. (2019). Interações medicamentosas de fitoterápicos utilizados no tratamento da insônia: uma breve revisão. Visão Acadêmica, 20(3).

Gelatti, G. T., de Oliveira, K. R., \& de Fátima Colet, C. (2016). Potenciais interações relacionadas ao uso de medicamentos, plantas medicinais e fitoterápicos em mulheres no período do climatério Potential drug interactions in relation with the use, medicine plants and herbal in premenopausal women period. Revista de Pesquisa Cuidado é Fundamental Online, 8(2), 4328-4346.

Lorenz, C., Klein, D., Colet, C., Amador, T. A., \& Heineck, I. (2016). O uso de plantas medicinais e fitoterápicos em usuários de varfarina no município de Ijuí/RS. Salão do Conhecimento.

Marchetti, R. C. H. (2015). Histórico de mudanças de produto: uma inovação para os processos de alterações pós-registro de medicamentos. Arca - Fiocruz.

Marliére, L. D., Ribeiro, A. Q., Brandão, M. D. G. L., Klein, C. H., \& Acurcio, F. D. A. (2008). Utilização de fitoterápicos por idosos: resultados de um inquérito domiciliar em Belo Horizonte (MG), Brasil. Revista Brasileira de Farmacognosia, 18, 754-760.

Neves, T. D. O., \& Vieira, T. R. (2019). Principais interações entre medicamentos e as plantas medicinais e/ou fitoterápicos. Repositório Uniube.

Nicácio, R. A. R., Pinto, G. F., da Silva, F. R. A., da Silva Santos, D. A., de Matos, M., \& Goulart, L. S. (2020). Potenciais interações entre medicamentos alopáticos e fitoterápicos/plantas medicinais no Município de Rondonópolis-MT. Revista de Ciências Médicas e Biológicas, 19(3), 417-422.

Paixão, J. A., Conceição, R. S., NETO, A. F. S., Neto, J. F. A., \& DOS SANTOS, U. S. (2016). Levantamento bibliográfico de plantas medicinais comercializadas em feiras da Bahia e suas interações medicamentosas. Revista Eletrônica de Farmácia, 13(2), 71-81.

Riboldi, L. S., \& Rigo, M. P. M. (2019). Análise do uso de plantas medicinais e medicamentos em habitantes do município de capitão/RS. Revista Destaques Acadêmicos, 11(3).

Sá, L. L. F., da Silva, H. V. A., Nogueira, N. C., de Oliveira, K. B. V., \& de Brito, M. D. R. M. (2018). Regulamentação de fitoterápicos no Brasil e perfil daqueles vendidos em uma farmácia de manipulação de Teresina-PI. Boletim Informativo Geum, 9(3), 1-9.

Silva, A.C, \& Rodrigues Leitão, JMS (2021). Interações medicamentosas associada a Ginkgo biloba L .: Revisão de literatura. Research, Society and Development , $10(6)$.

Silva, I. C. M., Júnior, E. S. C., \& Rivanor, R. L. D. C. (2018). Levantamento dos Fitoterápicos mais comercializados na cidade de Quixadá-Ce. III JornutriCisnce Faculdade de Quixadá.

Silva, N. C. S. (2016). Tudo que é natural não faz mal? Investigação sobre o uso de plantas medicinais e medicamentos fitoterápicos por idosos, na cidade de iapu-leste de Minas Gerais. ÚNICA Cadernos Acadêmicos, 2(1).

Silveira, P. F. D., Bandeira, M. A. M., \& Arrais, P. S. D. (2008). Farmacovigilância e reações adversas às plantas medicinais e fitoterápicos: uma realidade. Revista Brasileira de Farmacognosia, 18, 618-626.

Souza, J. D. M., \& SILVA, C. D. P. Chás e fitoterápicos indicados para distúrbios do sono, ansiedade e depressão, disponibilizados em estabelecimentos comerciais de São Caetano do Sul-SP. $21^{\circ}$ Congresso Nacional de Iniciação Científica.

Souza, M. T. D., Silva, M. D. D., \& Carvalho, R. D. (2010). Integrative review: what is it? How to do it?. Einstein (São Paulo), 8, 102-106. 